06.5;05.3

\title{
Электронно-пучковая кристаллизация тонких пленок аморфного субоксида кремния
}

\author{
(C) Е.А. Баранов ${ }^{1}$, В.О. Константинов ${ }^{1}$, В.Г. Щукин ${ }^{1}$, А.О. Замчий ${ }^{1,2}$, И.Е. Меркулова ${ }^{1,2}$, \\ Н.А. Лунёв ${ }^{1,2}$, В.А. Володин ${ }^{2,3}$ \\ ${ }^{1}$ Институт теплофиизики им. С.С. Кутателадзе СО РАН, Новосибирск, Россия \\ ${ }^{2}$ Новосибирский государственный университет, Новосибирск, Россия \\ ${ }^{3}$ Институт физики полупроводников им. А.В. Ржанова СО РАН, Новосибирск, Россия \\ E-mail: itpbaranov@gmail.com
}

Поступило в Редакцию 24 сентября 2020 г.

В окончательной редакции 30 ноября 2020 г.

Принято к публикации 6 декабря 2020г.

\begin{abstract}
Впервые получен поликристаллический кремний (poly-Si) в результате воздействия электронного пучка на пленки аморфного гидрогенизированного субоксида кремния со стехиометрическим коэффициентом $0.5\left(a-\mathrm{SiO}_{0.5}: \mathrm{H}\right)$ и толщиной $580 \mathrm{~nm}$. Ускоряющее напряжение электронного пучка составляло $2000 \mathrm{~V}$, а ток пучка - $100 \mathrm{~mA}$. Получены спектры комбинационного рассеяния света пленок кремния после отжига в зависимости от времени воздействия электронного пучка на исходный материал. Показано, что в результате отжига формируется поликристаллический кремний, напряжения в котором в зависимости от времени воздействия изменяются от сжатия до растяжения.
\end{abstract}

Ключевые слова: тонкие пленки субоксида кремния, электронно-пучковый отжиг, поликристаллический кремний.

DOI: 10.21883/PJTF.2021.06.50754.18560

Тонкие пленки поликристаллического кремния (poly-Si) широко используются в дисплеях с активной матрицей [1] и, кроме того, имеют хорошие перспективы применения в современных солнечных элементах [2] и тонкопленочных транзисторах на гибких подложках [3]. Отметим, что все эти приложения используют синтез поликристаллического кремния при низкой температуре, поскольку в качестве подложек применяются такие материалы, как стекло и пластик. Наиболее распространенным способом получения тонких пленок poly-Si на низкотемпературных подложках является кристаллизация пленок аморфного кремния $(a-\mathrm{Si})$. Среди методов кристаллизации, которые используют прямое воздействие на исходную пленку кремния, можно выделить лазерный отжиг (laser annealing) [3], импульсный фотонный отжиг (flash lamp annealing) [4], отжиг термоплазменной струей (thermal plasma jet) [5], электронно-пучковую кристаллизацию (electron beam crystallization) [6,7].

В настоящей работе получен поликристаллический кремний в результате электронно-пучкового отжига тонких пленок аморфного гидрогенизированного субоксида кремния $\left(a-\mathrm{SiO}_{x}: \mathrm{H}\right)$. Проведено исследование влияния времени экспозиции электронного пучка на напряжения в поликристаллическом кремнии.

Для проведения экспериментов по электронно-пучковому отжигу были получены образцы с тонкими пленками аморфного гидрогенизированного субоксида кремния на кварцевых (марка КУ-1, толщина $1 \mathrm{~mm}$ ) и кремниевых (пластина монокристаллического кремния КЭФ4.5, ориентация $\langle 100\rangle$, толщина $420 \mathrm{~nm}$ ) подложках размером $12 \times 18 \mathrm{~mm}$. Тонкие пленки $a-\mathrm{SiO}_{x}: \mathrm{H}$ были получены методом плазмохимического осаждения смеси $\mathrm{SiH}_{4}-\mathrm{O}_{2}$ из газовой фазы с использованием широкоапертурного источника с индуктивным высокочастотным возбуждением (13.56 MHz). Мощность высокочастотного излучения и температура подложки в процессе синтеза составляли $50 \mathrm{~W}$ и $150^{\circ} \mathrm{C}$ соответственно.

Отжиг пленок $a-\mathrm{SiO}_{x}: \mathrm{H}$ осуществлялся в вакуумной камере с давлением $10^{-6}$ mbar. C помощью электронной пушки на основе разряда с полым катодом генерировался электронный пучок [8]. Ускоряющее напряжение электронного пучка составляло $2000 \mathrm{~V}$, ток пучка 100 mА. Подложкодержатель с установленным на нем образцом размещался перпендикулярно электронному пучку. Время воздействия регулировалось включениемвыключением электронной пушки. Плотность тока на поверхности образца составила $15 \mathrm{~mA} / \mathrm{cm}^{2}$.

На изображении поперечного разреза исходного образца (рис. 1), сделанном на растровом электронном микроскопе (PЭМ) JEOL JSM-6700F, хорошо видна пленка $a-\mathrm{SiO}_{x}: \mathrm{H}$, толщина которой составила $580 \mathrm{~nm}$. Наблюдается характерная столбчатая структура пленки, обусловленная высокой концентрацией водорода. Столбцы с большим количеством вертикальных пустот начинаются от подложки и проходят вдоль всей пленки в направлении ее роста. Проведенные с помощью рентгеновской спектроскопии с дисперсией по энергии (EDS) измерения атомной концентрации кремния и кислорода в исходных пленках показали, что стехиометрический коэффициент (отношение атомной концентрации кислорода к атомной концентрации кремния в пленке) этих пленок равен 0.52 . 


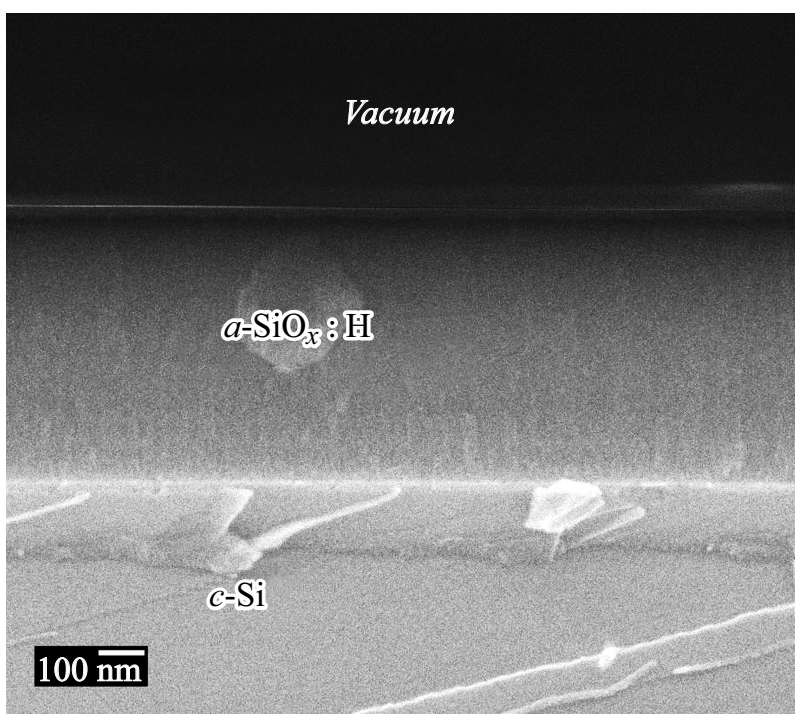

Рис. 1. РЭМ-изображение поперечного сечения тонкой пленки $a-\mathrm{SiO}_{x}: \mathrm{H}$ на пластине монокристаллического кремния.

Для исходных пленок $a-\mathrm{SiO}_{x}: \mathrm{H}$ были получены спектры пропускания в ИК-диапазоне (FTIR) на приборе Scimitar FTS 2000. На спектрах FTIR присутствуют полосы, соответствующие колебаниям атомных связей $\mathrm{Si}-\mathrm{H}$ и $\mathrm{O}-\mathrm{Si}-\mathrm{O}$ в пленке. Для определения концентрации водорода в пленках использовалось интегральное поглощение полосы на частоте $640 \mathrm{~cm}^{-1}$, которое соответствует веерному и маятниковому колебаниям связей $\mathrm{Si}-\mathrm{H}$ [9]. Для полученных пленок концентрация водорода составила 20 at.\%.

Спектры комбинационного рассеяния света (КРС) были получены при комнатной температуре в обратной геометрии рассеяния с использованием спектрометра T64000 фирмы Horiba Jobin Yvon. Возбуждение КРС осуществлялось на длине волны $514.5 \mathrm{~nm}$, и лазерный луч фокусировался в пучок диаметром менее $5 \mu \mathrm{m}$. Для предотвращения локального нагрева образцов и сдвига частот фононов вследствие ангармонизма мощность возбуждающего лазерного излучения выбиралась в диапазоне, в котором дальнейшее уменьшение мощности не приводило к изменению положения пика poly-Si.

Спектры КРС исходных пленок $a-\mathrm{SiO}_{x}: \mathrm{H}$ воспроизводят эффективную плотность колебательных состояний связей $\mathrm{Si}-\mathrm{Si}$ и характерны для аморфной структуры материала (см. вставку на рис. 2). Спектры комбинационного рассеяния света пленок субоксида кремния, полученные в зависимости от времени электроннопучкового отжига, представлены на рис. 2. На спектрах присутствует характерный пик поликристаллического кремния, что свидетельствует о том, что в результате электронно-пучкового отжига прошел процесс кристаллизации аморфного субоксида кремния с образованием poly-Si для времени воздействия электронного пучка 60,180 и $600 \mathrm{~s}$ (доза облучения 0.9, 2.7 и 9 $\mathrm{C} / \mathrm{cm}^{2}$ соответственно). Положение пика для времени отжи- га $60 \mathrm{~s}$ составило $518.4 \mathrm{~cm}^{-1}$, ширина на полувысоте $6.1 \mathrm{~cm}^{-1}$ согласно спектру, показанному на рис. 2. Для времени отжига $180 \mathrm{~s}$ положение пика poly-Si составило $519.7 \mathrm{~cm}^{-1}$, ширина на полувысоте $7.1 \mathrm{~cm}^{-1}$. С увеличением времени отжига до $600 \mathrm{~s}$ положение пика сместилось до значения $524.5 \mathrm{~cm}^{-1}$ при ширине на полувысоте $6.5 \mathrm{~cm}^{-1}$ (рис. 2). Следует отметить, что для пучка электронов с ускоряющим напряжением $1000 \mathrm{~V}$ и током $100 \mathrm{~mA}$ даже при времени отжига $600 \mathrm{~s}$ формирования poly-Si не произошло, а воздействие пучка электронов с ускоряющим напряжением $3000 \mathrm{~V}$ и аналогичным током привело к испарению тонкой пленки $a-\mathrm{SiO}_{x}: \mathrm{H}$ за $60 \mathrm{~s}[10]$.

Спектры КРС монокристаллического кремния (c-Si) содержат узкий пик при $520.6 \mathrm{~cm}^{-1}$ вследствие того, что в кристалле сохраняется квазиимпульс и в рассеянии активны только длинноволновые оптические фононы. В случае механических напряжений, возникающих из-за дефектов или несоответствия коэффициента термического расширения между подложкой и пленкой, пик от оптических фононов смещается [11] от положения $520.6 \mathrm{~cm}^{-1}$. Сжимающее напряжение приводит к сдвигу спектров комбинационного рассеяния выше $520.6 \mathrm{~cm}^{-1}$, в то время как растягивающее напряжение приводит к сдвигу спектров комбинационного рассеяния ниже $520.6 \mathrm{~cm}^{-1}$.

Используя значение смещения пика poly-Si, можно оценить значение напряжения согласно уравнению $[12,13]$ :

$$
\sigma[\mathrm{MPa}]=-250\left(\omega-\omega_{0}\right)\left[\mathrm{cm}^{-1}\right],
$$

где $\omega_{0}-$ положения пика $c$-Si без напряжений, a $\omega-$ положения пика напряженного poly-Si. Значения напряжения в poly-Si, полученные из спектров КРС, в зависимости от времени воздействия электронного пучка

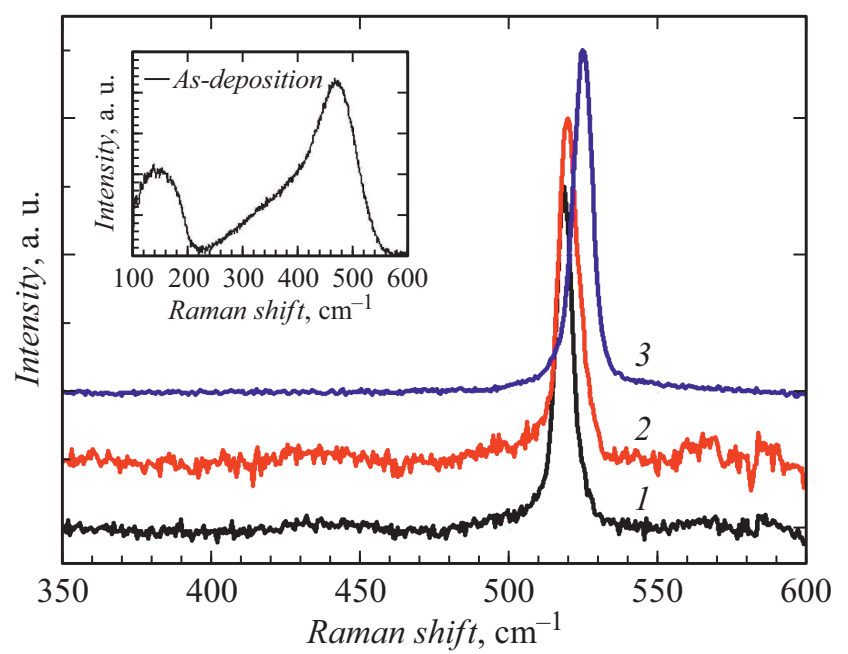

Рис. 2. Спектры КРС образцов, полученных в результате отжига электронным пучком с ускоряющим напряжением $2000 \mathrm{~V}$ и током $100 \mathrm{~mA}$, для времени воздействия 60 (1), 180 (2) и $600 \mathrm{~s}$ (3). На вставке приведен спектр КРС исходного образца. 


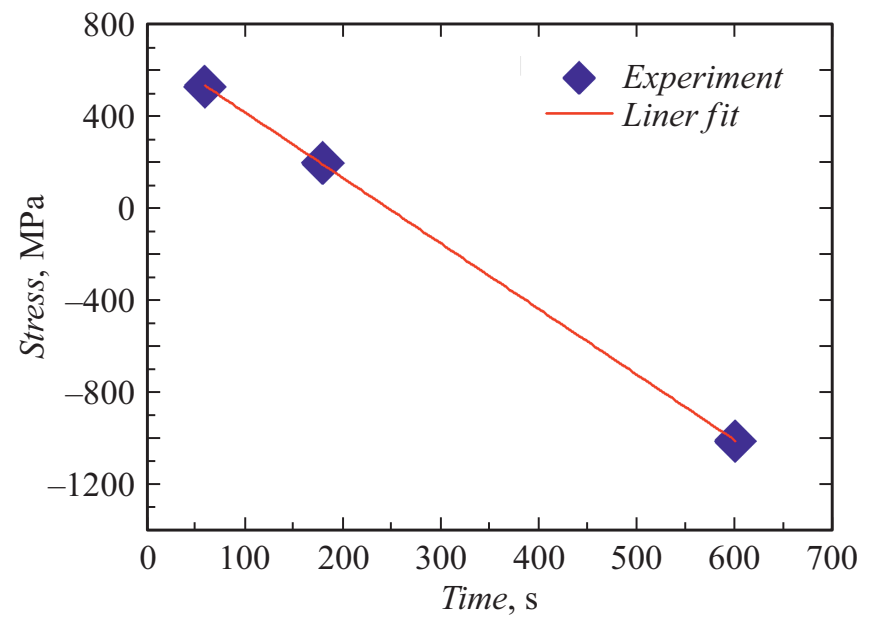

Рис. 3. Значения напряжения в пленках поликремния, полученные исходя из положения пиков КРС, в зависимости от времени воздействия электронного пучка.

показаны на рис. 3. Погрешность измерения составила порядка $170 \mathrm{MPa}$. Видно, что с увеличением времени воздействия напряжения в poly-Si изменяются от сжатия до растяжения и хорошо описываются линейной аппроксимацией.

Таким образом, в результате воздействия электронного пучка на пленки аморфного гидрогенизированного субоксида кремния впервые получен поликристаллический кремний (poly-Si). C помощью метода комбинационного рассеяния света для пленок кремния после отжига было показано, что напряжение в poly-Si изменяется в зависимости от времени экспозиции электронного пучка.

\section{Благодарности}

Авторы выражают благодарность ЦКП „ВТАН“ НГУ за предоставленное оборудование для регистрации спектров КРС.

\section{Финансирование работы}

Исследование выполнено при финансовой поддержке гранта Президента РФ (МК-638.2019.8) (синтез и диагностика пленок субоксида кремния) и Российского фонда фундаментальных исследований в рамках научного проекта № 19-08-00848 (электронно-пучковый отжиг).

\section{Конфликт интересов}

Авторы заявляют, что у них нет конфликта интересов.

\section{Список литературы}

[1] L. Wang, L. Sun, D. Han, Y. Wang, M. Chan, S. Zhang, J. Display Technol., 10 (4), 317 (2014).

DOI: $10.1109 /$ JDT.2014.2301554
[2] A. Rohatgi, K. Zhu, J. Tong, D.H. Kim, E. Reichmanis, B. Rounsaville, V. Prakash, Y.W. Ok, IEEE J. Photovolt., 10 (2), 417 (2020). DOI: 10.1109/JPHOTOV.2019.2963564

[3] Y. Do, D.Y. Jeong, S. Lee, S. Kang, S. Jang, J. Jang, Adv. Eng. Mater., 22 (5), 1901430 (2020). DOI: $10.1002 /$ adem. 201901430

[4] K. Kurata, K. Ohdaira, Jpn. J. Appl. Phys., 58 (SB), SBBF10 (2019). DOI: $10.7567 / 1347-4065 /$ aafb51

[5] R. Nakashima, R. Shin, H. Hanafusa, S. Higashi, Jpn. J. Appl. Phys., 56 (6S2), 06HE05 (2017). DOI: $10.7567 / J J A P .56 .06 H E 05$

[6] D. Amkreutz, J. Haschke, S. Kühnapfel, P. Sonntag, B. Rech, IEEE J. Photovolt., 4 (6), 1496 (2014). DOI: 10.1109/JPHOTOV.2014.2358799

[7] S.W. Lee, J.S. Kang, K.C. Park, J. Korean Phys. Soc., 68 (4), 528 (2016). DOI: 10.3938/jkps.68.52

[8] В.Г. Щукин, В.О. Константинов, В.С. Морозов, ЖТФ, 88 (6), 914 (2018). DOI: 10.21883/JTF.2018.06.46025.2544

[9] A.O. Zamchiy, E.A. Baranov, I.E. Merkulova, S.Y. Khmel, E.A. Maximovskiy, J. Non-Cryst. Solids, 518, 43 (2019). DOI: 10.1016/j.jnoncrysol.2019.05.015

[10] J. Kennedy, J. Leveneur, F. Fang, A. Markwitz, Nucl. Instrum. Meth. Phys. Res. B, 332, 421 (2014). DOI: 10.1016/j.nimb.2014.02.110

[11] C.W. Byun, S.W. Son, Y.W. Lee, H.M. Kang, S.A. Park, W.C. Lim, T. Li, S.K. Joo, J. Electrochem. Soc., 159 (4), J115 (2012). DOI: $10.1149 / 2.078204 j$ jes

[12] J.H. Park, H.Y. Kim, K.H. Seok, H.J. Chae, S.K. Lee, Y.H. Lee, Z. Kiaee, S.K. Joo, RSC Adv., 5 (69), 55909 (2015). DOI: $10.1039 / C 5 R A 09194 B$

[13] V.V. Bolotov, M.D. Efremov, V.A. Volodin, Thin Solid Films, 248 (2), 212 (1994). DOI: 10.1016/0040-6090(94)90013-2 